\title{
Novel Opt-coupling Transistor by LED and PD and its Application
}

\author{
Junichi Fujita, Daisuke Sato, Kensho Okamoto, Tetsuo Hattori \\ Department of Electronics and Information Engineering, Kagawa University, 2217-20 Hayashi-cho, \\ Takamatsu, Kagawa, 761-0396, Japan \\ E-mail: s12d451@stmail.eng.kagawa-u.ac.jp, \{oka, hattori\}@eng.kagawa-u.ac.jp
}

\begin{abstract}
This paper proposes a novel transistor-like device by the optical coupling of LED (Light Emitting Diode) and Photodiode. The device that we call "OCT: Optical Coupling Transistor" shows an amplification function if the Photodiode current by photo electromotive force is feed backed into the LED, in the same way as the case of common emitter circuit of the conventional Bipolar Junction Transistor. In this paper, we present the amplification principle and its applications by OCT such as audio amplifier and operational amplifier.
\end{abstract}

Keywords: Transistor, Thyristor, Opt-coupling, LED, Photodiode, Optical Coupling Transistor

\section{Introduction}

It is well known that the bipolar transistor is a semiconductor device capable of amplifying a small electric signal to a significant output level, e.g. of voltorder, in the case of analogue application.

However, as a matter of fact, the transistor itself generates neither a high voltage nor large current. Practically, the bipolar transistor behaves as a variable resistance element whose resistance value between the collector and the emitter can be controlled widely from near zero ohm to almost infinity ohm by a small input current, namely the Base current IB that is passed from the Base to the Emitter in NPN typed bipolar transistor. As the collector is connected to a resistor Rc in the common emitter transistor circuit, a constant direct current voltage VCC is applied to between RC and the Emitter. Under such a condition, if IB is passed from the base to the Emitter then RCE, which is the internal resistance between the Collector and the Emitter, will changes drastically. This causes the changes of the collector current IC and also VCE, the output voltage between Collector and Emitter.

One of the authors, Kensho Okamoto has noticed the behavior of the bipolar junction transistor as an electronically controllable resistor and so he thought that it might be possible to realize a new amplifier device by a system combining LED and silicon photodiode, or silicon solar cell, with positive feedback manner. In this paper, we call the device "OCT" that stands for Optical-Coupling typed Transistor.

Further-more, we show the fact that the OCT can become a thyristor-like switching device under a certain condition.

According to our literature search, there are only two similar researches ${ }^{1,2}$ in the world other than ours. However no practical devices were developed in their works.

\section{Transistor by Photo-Coupling}

The bipolar junction transistor was invented in 1948. As is well known, there are two types in the bipolar transistor, i.e. PNP and NPN transistors. Since the birth 
of transistor, the operation of such bipolar transistors have been explained by the behavior of electrons and positive holes in the Emitter(E), Base(B) and Collector(C) regions as illustrated in Fig.1 (a).

However, in 1989, B.J. Van Zeghbroeck et.al have proposed the notion of "Photon transport transistor". 1 And in 1996, W. N. Cheung and P. J. Edwards have theoretically shown the characteristics of class of optocoupler amplifier. ${ }^{2}$

On the other hand, as mentioned before, Okamoto who is one of the authors of this paper, also has found recently that the well-known common base transistor circuit in Fig.1 (a) can be realized by putting together an infrared light-emitting diode (IR-LED) and a $\mathrm{Si}$ photodiode (Si-PD) as shown in Fig.1 (b).

Then he made the quasi emitter common transistor circuit using IR-LED and Si-PD as shown in Fig.2 (b) in order to confirm whether the circuit operated as the real transistor amplifier shown in Fig.2 (a). ${ }^{3,4}$ Fig.3 (a) shows an example of the device which consists of an IR-LED and Si-PD. Fig.3 (b) shows picture of actual OCT module. Amplification factor $\alpha$, which is defined as IC/IE shown in Fig.3 (b), our first OCT was about 0.34 .

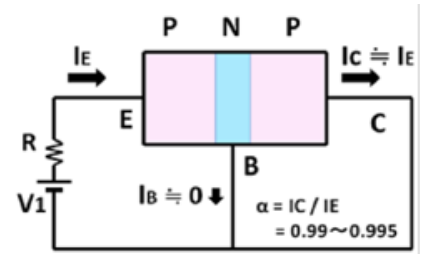

(a) Common base transistor.

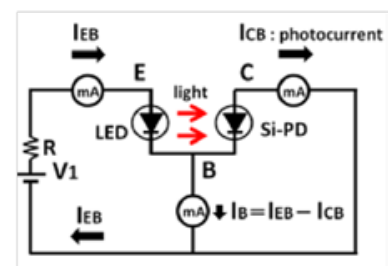

(b) OCT circuit.

Fig.1. Conventional bipolar transistor and OCT.,

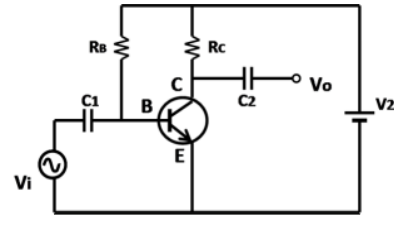

(a) Common emitter circuit.

\section{Fig.2. Common emitter circuits by the conventional bipolar} transistor and OCT.

However, afterwards we have obtained the fact that $\alpha$ value can reach to more than 0.9 in a best combination of LED and Si-PD. This is a great value because $\alpha \approx 0.99$ is of conventional transistor. According to the transistor theory, if $\alpha=0.9$, then it is possible to build an emitter-common transistor with $\beta=9$. This means that the LED-photodiode system can be used as an amplifier, in a similar way as the bipolar transistor.

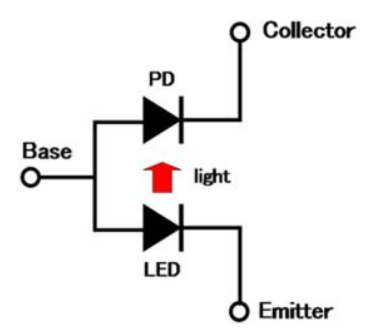

(a) Composition of OCT.

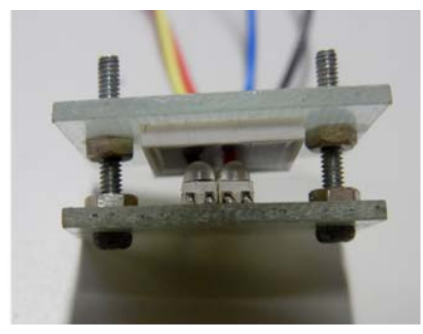

(b) Outlook of OCT.
Fig.3. The OCT that consists of Infrared LED and Si-PD. ${ }^{3,4}$

Fig.4 shows the amplification performance such that the quasi emitter common circuit using the OCT device works as a practical voltage amplifier. We can obtain a voltage gain as more than $40 \mathrm{~dB}$ by adjusting each value of RC, RB and V (the voltage of power source). However, it is difficult to make a power or a current amplifier using the OCT, because a normal photodiode does not generate photocurrent so large as $100 \mathrm{~mA}$, which is required to drive an $8 \mathrm{ohm}$ speaker.

Then, we used a $\mathrm{Si}$ solar cell module instead of a $\mathrm{Si}$ photodiode and succeeded in the development of a OCT power (or main) amplifier.

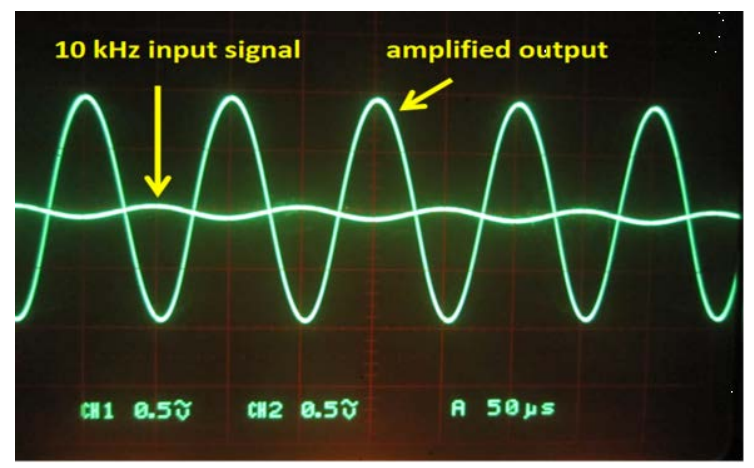

Fig.4. Amplification waveform using OCT in Fig.3.

Fig. 5 is an audio pre-main amplifier circuit using two sets of OCTs, and Fig. 6 is a hand-made practical OCT audio amplifier. We can enjoy beautiful and noise-free music sound using the audio system. ${ }^{3}$ 


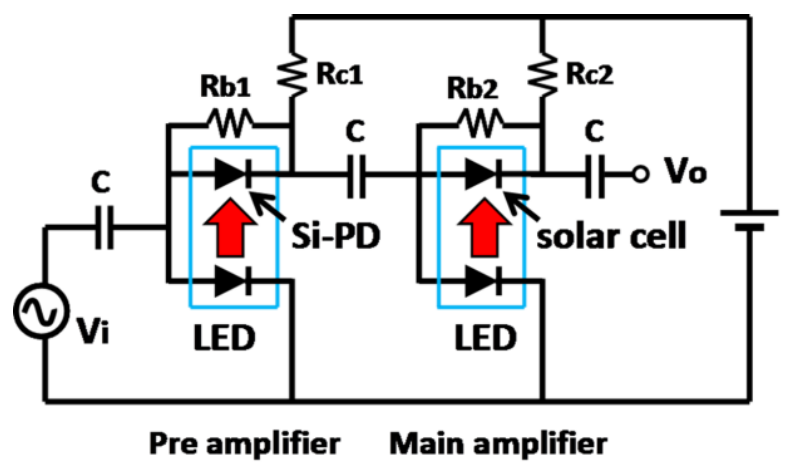

Fig.5. The audio amplifier circuit using OCTs. ${ }^{3}$

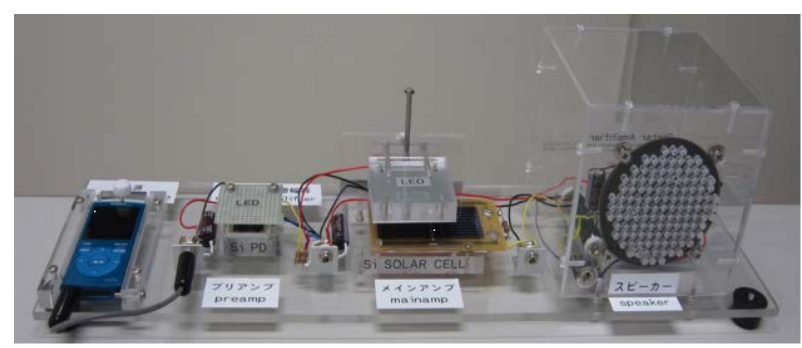

Fig.6. Outlook of the audio amplifier. ${ }^{3}$

\section{Amplification Function}

In this section, we explain about the amplification principle of the OCT. In the circuit of Fig.7, $I_{C}$ does not flow because the voltage direction of PD is reversely applied by the relation of connection to voltage source $\mathbf{V}$.

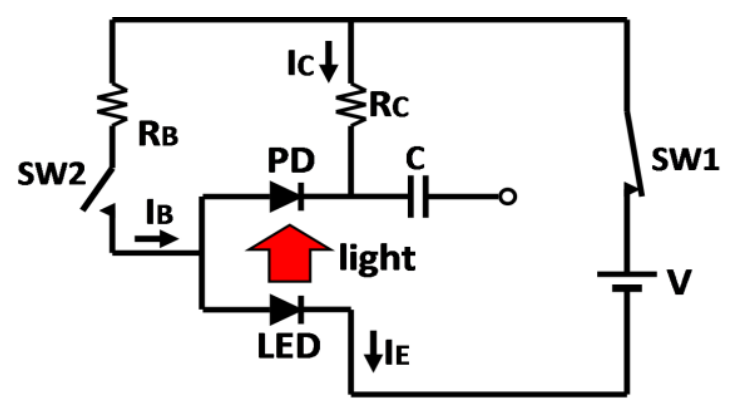

Fig.7. OCT circuit when SW1 is closed and SW2 is open. ${ }^{3,4}$

Therefore,

$$
I_{C}=I_{E}=\mathbf{0}\left(\text { when } I_{B}=0\right)
$$

As shown in Fig.8, Base-current $I_{B}$ flows through the LED connected between B to E when the switch SW2 is turned on. Hence,

$$
I_{E}=I_{B} \text { (when } I_{B} \text { flows) }
$$

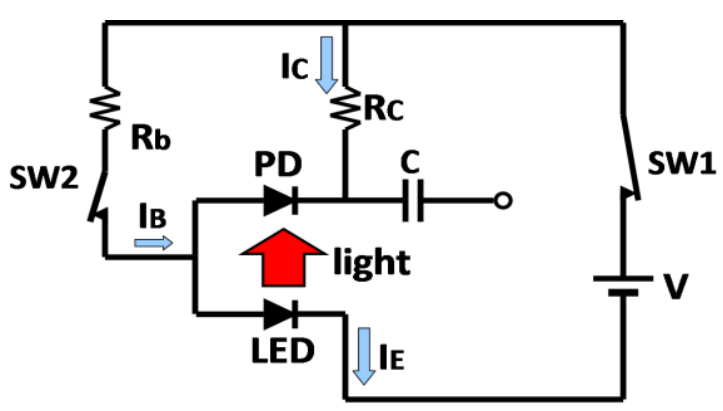

Fig.8. OCT circuit when SW1 and SW2 are closed. ${ }^{3,4}$

Then, the LED emits light and the LED light enters into the PD, because the LED and the PD is optically faced each other. Consequently, photovoltaic effect occurs in PD and the photocurrent $I_{C}$ generates and flows through RC and the PD. The $\alpha$ is defined as the ratio of the current flowing through the $\mathrm{PD}$ and the current owing through the LED as shown in Eq. (3).

$$
\alpha=\frac{I_{C}}{I_{E}}
$$

Therefore, $I_{C}$ is expressed as Eq. (4).

$$
I_{C}=\alpha \times I_{E}
$$

At this time, $I_{E}=I_{B}$, as shown in Eq.(2). Then, Eq.(4) can be expressed as Eq.(5).

$$
I_{C}=\alpha \times I_{B}
$$

At the next stage, the current $I_{C}$ flows into LED, then $I_{E}$ will increase by the current, and at this point, it becomes $I_{E}=I_{B}+I_{C}=I_{B}+\alpha I_{B}$. And then, the light intensity of the LED increases by $\alpha I_{B}$, so $I_{C}$ increases more by $\alpha\left(\alpha I_{B}\right)$. Accordingly, at this point, $I_{C}$ becomes the current as shown in Eq.(6).

$$
I_{C}=\alpha \cdot I_{B}+\alpha\left(\alpha \cdot I_{B}\right)
$$

In a similar way, we have the following equations.

$$
I_{C}=\alpha \cdot I_{B}+\alpha\left(\alpha \cdot I_{B}\right)+\alpha\left(\alpha^{2} \cdot I_{B}\right)+\cdots
$$




$$
I_{C}=I_{B}\left(\alpha+\alpha^{2}+\alpha^{3}+\cdots\right)
$$

In Eq.(8), the term $\left(\alpha+\alpha^{2}+\alpha^{3}+\cdots\right)$ is a well-known infinite progression and its sum becomes the value as shown in Eq.(9), if $\alpha$ is $0 \leq \alpha<1$.

$$
\left(\alpha+\alpha^{2}+\alpha^{3}+\cdots\right)=\frac{\alpha}{(1-\alpha)}
$$

Eventually, $I_{C}$ becomes as follows.

$$
I_{C}=\frac{\alpha I_{B}}{(1-\alpha)}
$$

The ratio of $I_{B}$ and $I_{C}$ is as shown in Eq.(11).

$$
\beta=\frac{I_{C}}{I_{B}}=\frac{\alpha}{(1-\alpha)}
$$

In Eq.(10), if $\alpha=0.5$ then $\beta=0.5$. However, if $\alpha=0.9$ then $\beta=9$. If $\alpha$ exceeds $0.9, \beta$ enhances greatly e.g. $\alpha=$ $0.99, \beta=99$.

On other hand, in case of $\alpha \geq 1$,

$$
I_{C}=+\infty
$$

In this case, as a matter of fact, the suitable resistance Rc suppresses the increase of the current $I_{C}$, and so the $I_{C}$ becomes stable. In this stable situation, even when the switch SW2 is turned off, the current $I_{C}$ continues to flow at the same value. This seems that the OCT can become a thyristor-like device.

\section{Variations}

\subsection{Other combination}

Fig.9 shows the measurement circuit of quasi common base circuit using OCT. The light intensity and the wavelength of LED and photocurrent of PD are very important in OCT. As shown in Fig.10, light emission wavelength of the LED $(940 \mathrm{~nm})$ and light reception of the PD $(960 \mathrm{~nm})$ are almost identical. For this reason, we were using only infrared LED, looking for the best optcoupling conditions.

However, we have tried to make an OCT using high luminous efficiency red LED as another combination of photo-coupling (Fig.11). The electrical characteristic of this OCT was much better than we thought.

The amplification factor $\alpha$ using OCT that consists of IR-LED and PD as shown in Fig.12. The $\alpha$ significantly varies depending on the LED current ILED when ILED less than about 200mA.

Fig.13 shows the $\alpha$ using OCT consists of red-LED and PD. It is stable when the ILED is greater than a few $10 \mathrm{~mA}$.

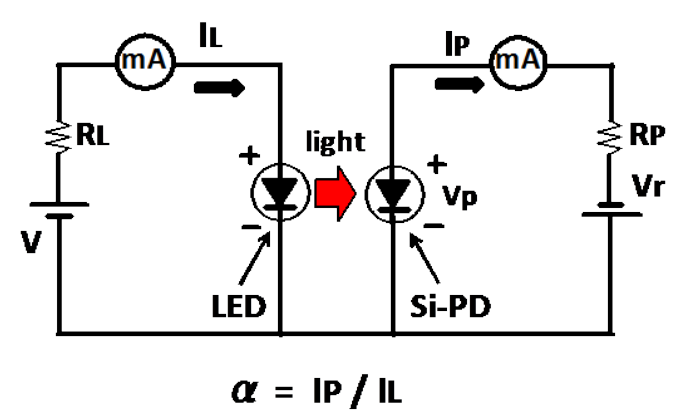

Fig.9. quasi common base circuit using OCT.

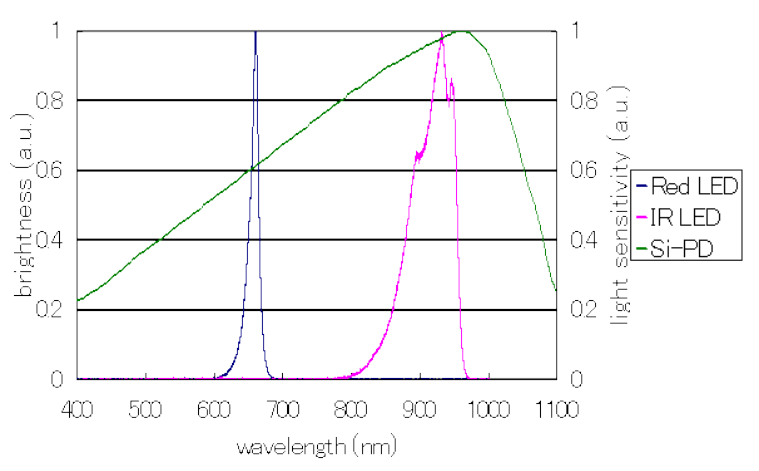

Fig.10. Spectrum of light emission and light reception.

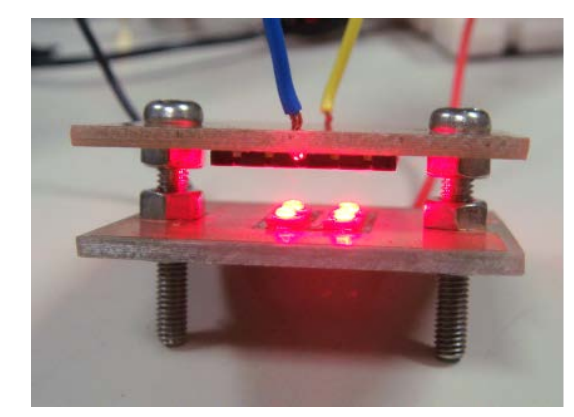

Fig.11. The OCT that consists of Red LED and Si-PD. 


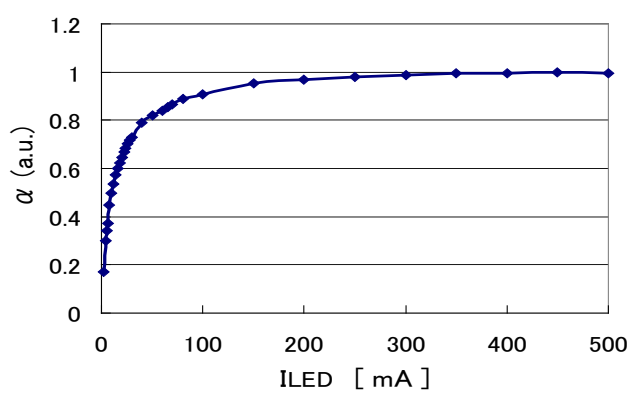

Fig.12. The $\alpha$ using OCT that consists of IR-LED and Si-PD.

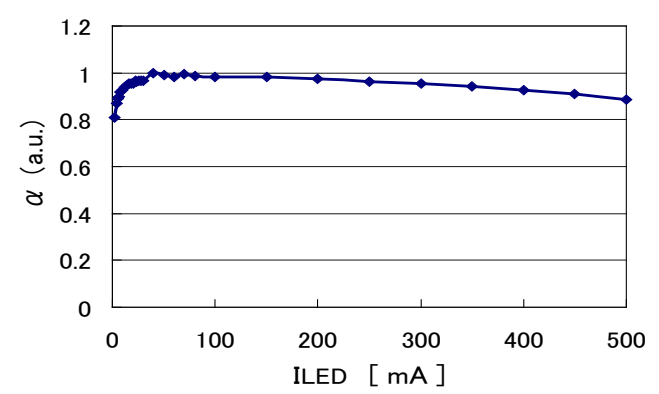

Fig.13. The $\alpha$ using OCT that consists of Red LED and Si-PD.

\subsection{Operational amplifier}

We have also made an operational amplifier using OCT module as shown in Fig.14. Moreover, we have confirmed that the Operational Amplifier in Fig.14 works as well as the conventional operational amplifier (Fig.15).

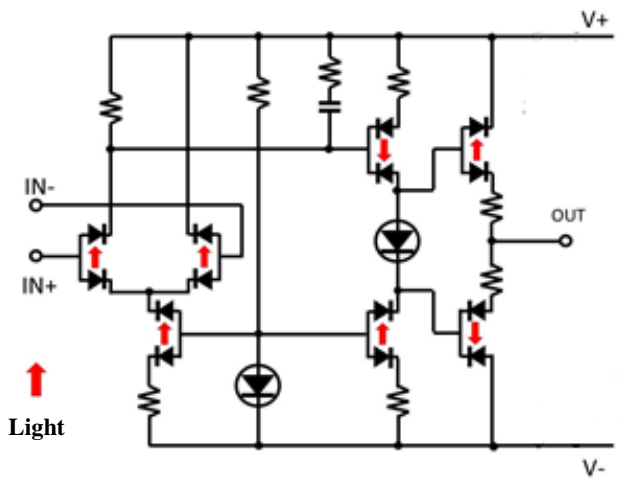

Fig.14. Operational amplifier using OCTs.

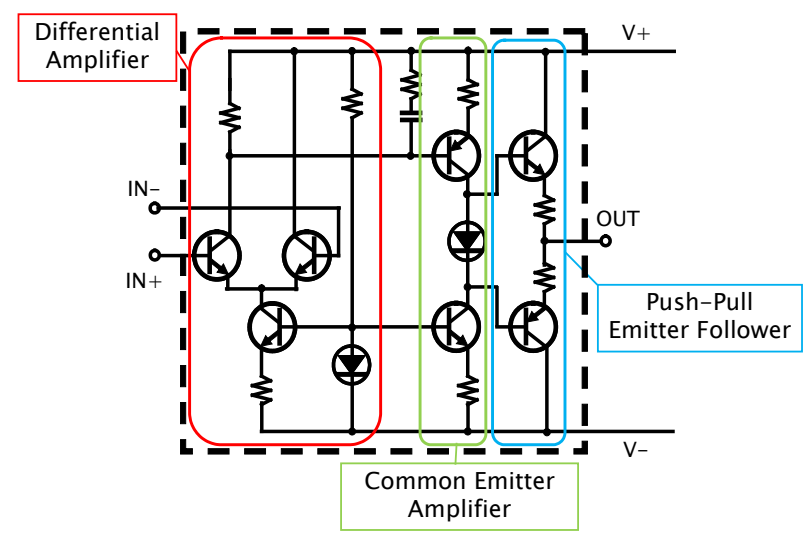

Fig.15. Operational Amplifier by the conventional bipolar transistors.

\section{Conclusion}

We have presented a novel transistor OCT by optcoupling of LED and PD. There are still rooms for improvement in realizing efficient transistors by the coupling. However, there is a high possibility that, the OCT that we have proposed in this paper, can be applied to develop a various kinds of practical devices, not only the power transistors such as thyristor, but also switching devices. We consider that the OCT will be very promising in the future.

\section{References}

1. B.J. Van Zeghbroeck, Ch. Herder, H. P. Meiner and W. Walter, "Photon transport transistor, in Proc. of 1989 IEEE International Electron Devices Meeting (IEDM), (IEEE, 1989) pp.543-546.

2. W. N. Cheung and P. J. Edwards, Characteristics of a Class of New Opto-Coupler Amplifiers with Positive Feedback, IEEE Journal of Quantum Electronics 32(3) (1996) 502-506.

3. J. Fujita, T. Hattori, K. Okamoto, Novel Audio Amplifier System Using Opt-coupling of LED and PD, in Proc. of 2013 International Conference on Biometrics and Kansei Engineering (ICBAKE2013), (IEEE, 2013), pp.301-304.

4. J. Fujita, K. Okamoto, T. Hattori, Development of novel transistor and thyristor composed of LED and photodiode, Japanese Journal of Applied Physics, 53(2014) (05FB181)-(05FB18-6). 